\title{
Biomarker to distinguish hepatocellular carcinoma from cholangiocarcinoma by serum a disintegrin and metalloprotease 12
}

\author{
Jureerut Daduang ${ }^{1}$, Temduang Limpaiboon¹, Sakda Daduang²
}

1Department of Clinical Chemistry, Centre for Research and Development of Medical Diagnostic Laboratories, Faculty of Associated Medical Sciences, Khon Kaen University, Thailand

2Department of Biochemistry, Faculty of Science, Khon Kaen University, Thailand

Submitted: 9 November 2010

Accepted: 8 August 2011

Arch Med Sci 2011; 7, 6: 1013-1016

DOI: 10.5114/AOMS.2011.26613

Copyright $\odot 2010$ Termedia \& Banach

\author{
Correspondence author: \\ Dr. Jureerut Daduang \\ Centre for Research and \\ Development of Medical \\ Diagnostic Laboratories \\ Faculty of Associated \\ Medical Sciences \\ Khon Kaen University \\ Khon Kaen, 40002, Thailand \\ Phone/fax: 66-43-202088 \\ E-mail: jurpoo@kku.ac.th
}

\begin{abstract}
Introduction: The "a distintegrin and metalloprotease" (ADAM) family contributes to regulation of the cell-cell and cell-matrix interaction that are critical determinants of malignancy. It also plays important roles in the degradation of the basement membrane during tumor invasion. To evaluate a distinguishing biomarker for hepatocellular carcinoma from cholangiocarcinoma, a disintegrin and metalloprotease 12 (ADAM12) level was determined.

Material and methods: The indirect ELISA and Western blot analysis for quantification of ADAM12 level in serum was developed. The subjects were 218 histologically confirmed cases, 128 with intrahepatic cholangiocarcinoma, 30 with hepatocellular carcinoma and 60 healthy people.

Results: The ability of test was verified using an analysis of Receiver Operating Characteristic (ROC) curve. The mean value of serum ADAM 12 in hepatocellular carcinoma was significantly higher than cholangiocarcinoma and healthy people $(p=0.001)$. The AUC for control vs. HCC was 0.826 while for controls vs. CC was 0.679 . The results showed that a disintegrin and metalloprotease 12 for hepatocellular carcinoma had better specificity $(77.4 \%)$ than for cholangiocarcinoma (64.5\%). The serum a disintegrin and metalloprotease 12 level was also found to inversely correlate with overall survival $(p=0.02)$.

Conclusions: A disintegrin and metalloprotease 12 would be most useful as an adjunct biomarker for distinguishing hepatocellular carcinoma from cholangiocarcinoma.
\end{abstract}

Key words: ADAM12 protein, cholangiocarcinoma, hepatocellular carcinoma, A disintegrin and metalloproteases.

\section{Introduction}

The two major subtypes of primary liver cancer are hepatocellular carcinoma (HCC) and cholangiocarcinoma (CC). Unlike HCC, CC contains a significant amount of fibrous stroma and subsequently low incidence of metastases of primary cancer. In fact, HCC patients have a similar clinical presentation as CC, such as upper abdominal pain, dyspepsia and fatigue. Therefore, initial misdiagnosis between HCC and intrahepatic or peripheral cholangiocarcinoma is commonly found, even though excellent studies have revealed that the serum markers for HCC and CC screening diagnostics are $\alpha$-fetoprotein [1] and MUC5AC [2], respectively. However, differen- 
tiation of HCC from CC by new serum markers is still a challenging study. A disintegrin and metalloproteases (ADAMs) are membrane-anchored cell surface glycoproteins. To date, more than 30 ADAMs have been identified. ADAM12 belongs to the large family of ADAMs and possesses extracellular metalloprotease and cell-binding functions, as well as intracellular signalling capacities. Interest in ADAM12 has increased recently because its expression is related to a variety of human cancers such as breast [3], bladder [4], lung [5], and liver [6, 7]. ADAMs mediated cancer cell proliferation and progression may be connected with their structures, which have the potential to possess protease, adhesion, fusion, and signalling functions $[8,9]$. Our report represents the first study of the ADAM12 level in serum samples taken from patients with chronic liver disease of different aetiologies. In this study, we developed an enzyme-linked immunosorbent assay (ELISA) for the quantification of ADAM12. 218 archived serum samples were tested.

\section{Material and methods}

\section{Patients}

Samples were obtained from patients with intrahepatic cholangiocarcinoma and hepatocellular carcinoma who had undergone surgery at Srinagarind Hospital, Faculty of Medicine, Khon Kaen University, Khon Kaen, Thailand. Informed consent was obtained from each patient. The Human Research Ethics Committee of Khon Kaen University approved the protocol (HE500602). Preoperative serum samples were obtained from 128 patients with CC, and 30 with HCC. The group of healthy adult controls consisted of 60 volunteers with the

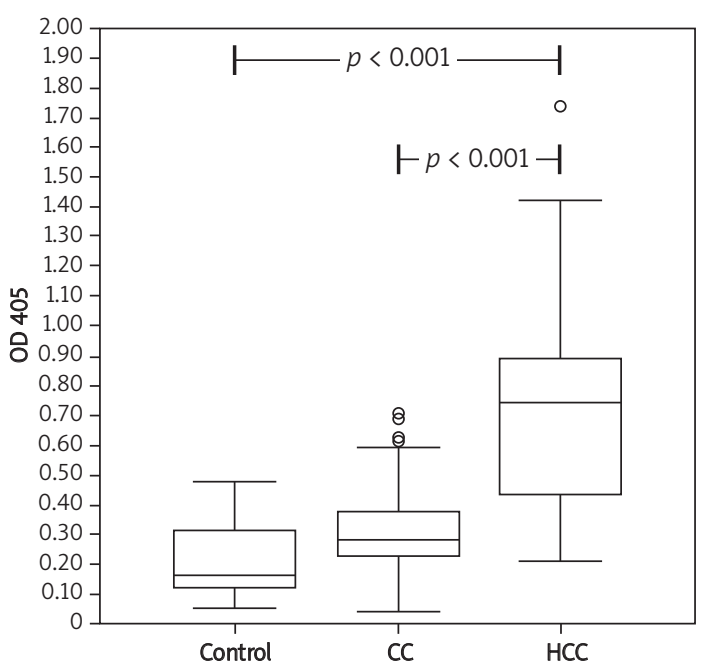

Figure 1. Indirect ELISA of serum ADAM12 in healthy control $(N=60), C C(N=128)$, and HCC $(N=30)$ Box $-95 \%$ confidence interval of ADAM12 level; line - mean of 3 independent experiments; asterisk $-p<0.05$ vs. control same average age as the CC group. All serum samples were stored at $-70^{\circ} \mathrm{C}$ until determined by ELISA and Western blot analysis using specific antibody.

\section{Enzyme-linked immunosorbent assay (ELISA)}

Serum samples were prepared in triplicate at $50 \mu \mathrm{l} /$ well, slightly modified from the previous report [10]. Each well of the polystyrene microtitre plate was coated with $1 \mu \mathrm{g} / 50 \mu \mathrm{l}$ serum in $50 \mathrm{mM}$ carbonate buffer, $\mathrm{pH}$ 9.5, and incubated overnight at $4^{\circ} \mathrm{C}$. The wells were washed with Tris-buffered saline (TBS, in mM: 10 Tris- $\mathrm{HCl}, \mathrm{pH} 8.0$ and $150 \mathrm{NaCl}$ ) containing $0.05 \%$ Tween 20 (TBST) and were blocked with $5 \%$ skimmed milk in TBST for 60 min at $37^{\circ} \mathrm{C}$. After three washes, specific primary antibody (Rabbit anti-human ADAM12; Catalogue no. A2601; Sigma), diluted in TBST, was incubated for $60 \mathrm{~min}$ at $37^{\circ} \mathrm{C}$. The wells were washed with TBST and incubated with secondary antibody (Goat antirabbit Igs conjugated alkaline phosphatase) diluted in TBST. Following incubation at $37^{\circ} \mathrm{C}$ for $60 \mathrm{~min}$, the wells were washed with TBST and TBS and visualized using freshly prepared chromogenic substrate $(1 \mathrm{mg} / \mathrm{ml}$ of $p$-nitrophenyl phosphate, $100 \mathrm{mM}$ Tris- $\mathrm{HCl}, \mathrm{pH}$ 9.5, $100 \mathrm{mM} \mathrm{NaCl}$ and $50 \mathrm{mM} \mathrm{MgCl}$ ). The absorbance was measured at $405 \mathrm{~nm}\left(\mathrm{~A}_{405}\right)$ by an ELISA plate reader.

\section{Western blot analysis}

Equal amounts of proteins (20 mg) were separated by $12 \%$ SDS-PAGE under reducing conditions. Resolved proteins were electrophoretically transferred to nitrocellulose membranes. The membranes were blocked and incubated with $1 \mathrm{mg} / \mathrm{ml}$ of specific primary antibody (Rabbit anti-human ADAM12; Catalogue no. A2601; Sigma). The secondary antibody (Goat anti-rabbit Igs conjugated alkaline phosphatase) was subsequently incubated and then visualized using an Alkaline Phosphatase Conjugated Substrate Kit following the manual protocol. Band intensities were analysed with the ImageJ program.

\section{Statistical analysis}

Mean levels of ADAM12 were compared between cancers and control groups using one-way ANOVA. The ability of serum ADAM12 to differentiate among CC, HCC and control was utilized to plot all pairs of true positives and false positives at various cut-off levels. To evaluate the diagnostic accuracy of ADAM12, receiver operating characteristic (ROC) curve and the area under the curve (AUC) were analysed. In addition, the survival curves were calculated using Kaplan-Meier and log rank tests. The result was considered statistically significant when $p<0.05$. All statistical analysis was performed using SPSS (version 15.0). 


\section{Results}

\section{Serum ADAM12 level}

Serum level of ADAM12 is shown in Figure 1. Mean levels in HCC (mean $\pm \mathrm{SD}=0.730 \pm 0.278$, $n=30)$ were significantly higher $(p<0.001)$ than in $C C$ (mean $\pm S D=0.313 \pm 0.130, n=128)$ and healthy controls (mean $\pm \mathrm{SD}=0.209 \pm 0.122, n=60$ ). We also examined the serum ADAM12 by Western blotting. The majority of samples from cancer patients, especially in the HCC group, were strongly significantly positive for ADAM12, in contrast to the normal serum samples (Figures $2 \mathrm{~A}$ and $2 \mathrm{~B}$ ). The result was similar to the ELISA, indicating that the developed technique is reliable.

\section{Receiver operating characteristic curves}

Based on the ROC curve analysis, the ADAM12 OD cut-off for control vs. HCC was 0.343 yield with AUC $=0.826$ while for controls vs. $C C$ it was 0.265 yield with $A \cup C=0.679$ (Figure 3 ). We also calculated the sensitivity, specificity, positive likelihood ratio (PLR), and negative likelihood ratio (NLR) of the marker. Our results showed that when comparing type of tumour, ADAM12 for HCC had better specificity and PLR (77.4\% and 3.51, respectively) than for CC (64.5\% and 1.25, respectively).

\section{Serum ADAM12 and survival rate}

Cumulative survival was compared between 60 patients with HCC. Serum ADAM12 in HCC was significantly correlated with the overall survival $(p=0.02)$, indicating that a high serum level of ADAM12 had a significantly poorer prognosis.

\section{Discussion}

As shown in Figure 1, the serum ADAM12 levels were significantly higher in HCC than in CC. Similarly, in vivo, Le Pabic et al. demonstrated that the steady state of ADAM12 mRNA levels was nearly undetectable in both normal livers and benign tumours and increased in HCC up to 6-fold and in liver metastases from colonic carcinomas up to 60-fold [6]. Roy et al. demonstrated that ADAM12 cleaves various extracellular matrix protein molecules including gelatin, type IV collagen and fibronectin, suggesting that the role of ADAM12 in cancer development and metastasis may be linked to their proteinase activity [3]. Schwettmann et al. suggested that ADAMs are differentially expressed in the liver. They assumed that ADAM-9, -TS1 23 and -TS2 play a crucial role in ECM remodelling during fibrotic processes in the liver [7] with the same as MMP2 and MMP9 in other chronic disease [11]. Based on tumour cell behaviour, there is mostly no distant metastasis and regional lymph node metastasis is common in CC while vascular invasion is less

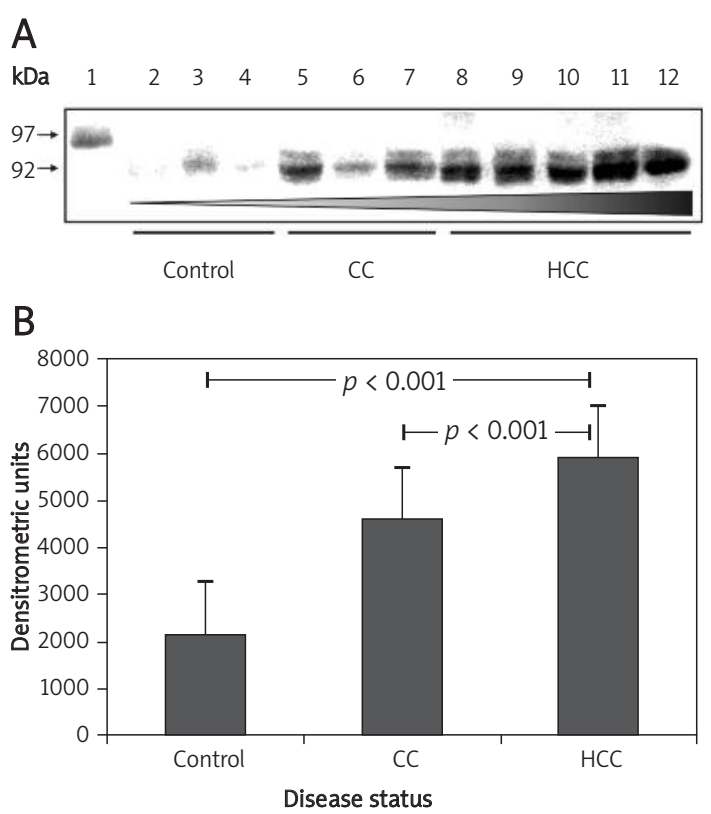

Figure 2. Western blot analysis of serum protein. A - Representative serum samples (20 mg of total protein per sample) from controls, CC and HCC were probed with ADAM12-specific. The bands of serum controls (lanes 2-4), CC (lanes 5-7) and HCC (lanes 8-12) are shown. B - Histogram of densitometry of the immunoblots indicates the increase in mean levels of serum ADAM12 with diseases status. The error bars denote standard errors

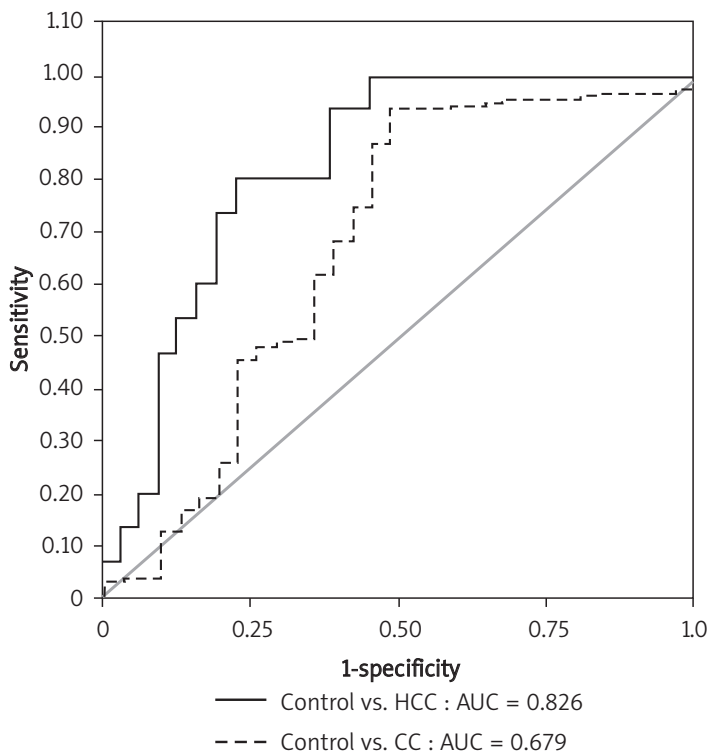

Figure 3. The ROC curve of serum ADAM12 in distinguishing between control subjects and patients with HCC and CC. The ROC curve of serum ADAM12 in control vs. HCC (AUC $=0.826$ ) was located closer to the theoretical $100 \%$ sensitivity and specificity values than in control vs. CC (AUC $=0.679)$

than in HCC. In this study, ADAM12 can discriminate CC patients from those with HCC who have similar clinical presentations. Thus, serum ADAM12 would 
be most useful as an adjunct biomarker for distinguishing HCC from CC.

\section{Acknowledgments}

This work was supported by the Thailand Research Fund (Grant no. MRG4980138) and the Centre for Research and Development of Medical Diagnostic Laboratories, Faculty of Associated Medical Sciences, Khon Kaen University, Thailand. We would like to greatly thank the Liver Fluke and Cholangiocarcinoma Research Center, Khon Kaen University, for all samples and clinical data.

\section{References}

1. Trevisani F, D'Intino PE, Morselli-Labate AM, et al. Serum alpha-fetoprotein for diagnosis of hepatocellular carcinoma in patients with chronic liver disease: influence of HBsAg and anti-HCV status. J Hepatol 2001; 34: 570-5.

2. Bamrungphon W, Prempracha N, Bunchu N, et al. A new mucin antibody/enzyme-linked lectin-sandwich assay of serum MUC5AC mucin for the diagnosis of cholangiocarcinoma. Cancer Lett 2007; 247: 301-8.

3. Roy R, Wewer UM, Zurakowski D, Pories SE, Moses MA. ADAM 12 cleaves extracellular matrix proteins and correlates with cancer status and stage. J Biol Chem 2004; 279: 51323-30.

4. Frohlich C, Albrechtsen R, Dyrskjot L, Rudkjaer L, Orntoft TF, Wewer UM. Molecular profiling of ADAM12 in human bladder cancer. Clin Cancer Res 2006; 12: 735-8.

5. Rocks N, Paulissen G, Quesada Calvo F, et al. Expression of a disintegrin and metalloprotease (ADAM and ADAMTS) enzymes in human non-small-cell lung carcinomas (NSCLC). Br J Cancer 2006; 94: 724-30.

6. Le Pabic H, Bonnier D, Wewer UM, et al. ADAM12 in human liver cancers. TGF-beta-regulated expression in stellate cells is associated with matrix remodeling. Hepatology 2003; 37: 1056-66.

7. Schwettmann L, Wehmeier M, Jokovic D, et al. J Hepatol 2008; 49: 243-50.

8. Schlondorff J, Blobel CP. Metalloprotease-disintegrins: modular proteins capable of promoting cell-cell interactions and triggering signals by protein-ectodomain shedding. J Cell Sci 1999; 112: 3603-17.

9. Black RA, White JM. ADAMs: focus on the protease domain. Curr Opin Cell Biol 1998; 10: 654-9.

10. Uawonggul N, Chaveerach A, Thammasirirak S, Arkaravichien T, Chuachan C, Daduang S. Screening of plants acting against Heterometrus laoticus scorpion venom activity on fibroblast cell lysis. J Ethnopharmacol 2006; 103: 201-7.

11. Kłysik AB, Kik JN, Hrabec Z, Goś R, Hrabec E. Intraocular matrix metalloproteinase 2 and 9 in patients with diabetes mellitus with and without diabetic retinopathy. Arch Med Sci 2010; 6: 375-81. 